\title{
SO2426 is a positive regulator of siderophore expression in Shewanella oneidensis MR-1
}

\author{
Kristene L Henne ${ }^{1 *}$, Xiu-Feng Wan ${ }^{2}$, Wei Wei ${ }^{1}$ and Dorothea K Thompson ${ }^{1,3^{*}}$
}

\begin{abstract}
Background: The Shewanella oneidensis MR-1 genome encodes a predicted orphan DNA-binding response regulator, SO2426. Previous studies with a SO2426-deficient MR-1 strain suggested a putative functional role for SO2426 in the regulation of iron acquisition genes, in particular, the siderophore (hydroxamate) biosynthesis operon s03030-3031-3032. To further investigate the functional role of SO2426 in iron homeostasis, we employed computational strategies to identify putative gene targets of $\mathrm{SO} 2426$ regulation and biochemical approaches to validate the participation of SO2426 in the control of siderophore biosynthesis in S. oneidensis MR-1.
\end{abstract}

Results: In silico prediction analyses revealed a single 14-bp consensus motif consisting of two tandem conserved pentamers ( $5^{\prime}$-CAAAA- $3^{\prime}$ ) in the upstream regulatory regions of 46 genes, which were shown previously to be significantly down-regulated in a so2426 deletion mutant. These genes included s03030 and so3032, members of an annotated siderophore biosynthetic operon in MR-1. Electrophoretic mobility shift assays demonstrated that the SO2426 protein binds to its motif in the operator region of s03030. A "short" form of SO2426, beginning with a methionine at position 11 (M11) of the originally annotated coding sequence for SO2426, was also functional in binding to its consensus motif, confirming previous 5' RACE results that suggested that amino acid M11 is the actual translation start codon for SO2426. Alignment of SO2426 orthologs from all sequenced Shewanella spp. showed a high degree of sequence conservation beginning at M11, in addition to conservation of a putative aspartyl phosphorylation residue and the helix-turn-helix $(\mathrm{HTH})$ DNA-binding domain. Finally, the so2426 deletion mutant was unable to synthesize siderophores at wild-type rates upon exposure to the iron chelator 2,2'-dipyridyl.

Conclusions: Collectively, these data support the functional characterization of SO2426 as a positive regulator of siderophore-mediated iron acquisition and provide the first insight into a coordinate program of multiple regulatory schemes controlling iron homeostasis in S. oneidensis MR-1.

\section{Background}

Bacteria sense and respond to environmental stimuli primarily through signal transduction pathways, in which the canonical mechanism employs a sensor histidine kinase that interacts with a DNA-binding response regulator to activate or repress specific gene transcription $[1,2]$. Some cellular processes have been shown to be controlled by orphan response regulators or one-component signalling systems, in which a cognate sensor kinase has not been elucidated [3]. Orphan response regulators have been shown to be involved in the regulation of motility and chemotaxis [4], growth-phase-dependent

\footnotetext{
*Correspondence: khenne@anl.gov; dthomps4@utk.edu

'Department of Biological Sciences, Purdue University, West Lafayette, IN 47907, USA

Full list of author information is available at the end of the article
}

responses [5,6], virulence [7], iron transport [8] and oxidative stress responses $[8,9]$. For instance, one well-characterized regulon that appears to be controlled by an orphan response regulator in S. oneidensis MR-1 is the ArcA regulon, which regulates the cellular response to aerobic and anaerobic respiratory conditions [10]. The distinguishing feature of ArcA in comparison to the analogous system in Escherichia coli is that there does not seem to be a cognate sensor kinase, ArcB, in S. oneidensis [10], suggesting that $S$. oneidensis ArcA may be an orphan response regulator.

Our previous work suggested that a putative orphan response regulator, $\mathrm{SO} 2426$, in S. oneidensis MR-1 may be an integral member of a metal-responsive regulon governing the up-regulation of genes involved in iron uptake and homeostasis in response to metal stress. The
C Biomed Central

() 2011 Henne et al; licensee BioMed Central Ltd. This is an Open Access article distributed under the terms of the Creative Commons Attribution License (http://creativecommons.org/licenses/by/2.0), which permits unrestricted use, distribution, and reproduction in any medium, provided the original work is properly cited. 
ferric iron uptake regulator (Fur) is the predominant mechanism by which bacteria regulate iron homeostasis [11]. Evidence suggests an additional iron responsive network regulated by SO2426 in S. oneidensis MR-1. Upregulation of $\mathrm{SO} 2426$ at both the protein and transcript levels in response to iron and acid stress has been observed in a $\Delta$ fur mutant strain of MR-1 [12-14]. Our previous studies investigating the transcriptomic and proteomic response of $S$. oneidensis to chromate challenge further revealed enhanced expression of so2426 under chromate stress $[15,16]$. In a so2426 deletion mutant, genes involved in iron acquisition and homeostasis such as the so3030-3031-3032 operon, which encodes siderophore biosynthesis genes, were consistently downregulated at high levels in the deletion mutant. Iron acquisition and storage systems are commonly up-regulated when bacteria are subjected to conditions of metal stress (e.g., chromate), and a link between iron transport and heavy metal sensitivity has been suggested [15,17]. It is possible that sequestration of iron prevents redox cycling between ferrous iron and chromate, which can lead to reactive intermediates and oxidative stress $[18,19]$. A consequence of this may be deficient intracellular iron concentrations that could inhibit growth. A cyclical response would ensue, resulting in up-regulation of iron uptake genes such as those involved in siderophore biosynthesis, which is similar to what has been demonstrated for $S$. oneidensis in response to chromate stress $[15,16,20]$.

The aim of the present study was to examine the function of the uncharacterized SO2426 response regulator within the context of siderophore biosynthesis. We used a bioinformatics approach to map putative SO2426-binding domains and biochemical assays to demonstrate the binding of SO2426 to predicted recognition sites. Electrophoretic mobility shift assays showed that a recombinant SO2426 protein binds to a putative SO2426 motif that exists within the operator region of the so3030-3031-3032 operon. Siderophore detection assays further showed a diminished capacity of the $\Delta s o 2426$ mutant strain to produce siderophores, particularly in the presence of the iron chelator 2,2'-dipyridyl. Based on the identification of a Fur-binding motif upstream of the predicted SO2426-binding site within the operator region of the so3030-3031-3032 operon, we postulate that there are likely multiple levels of regulation operating in S. oneidensis MR-1 to precisely adjust intracellular iron levels in response to cellular needs. These intricate control mechanisms appear to involve Fur-mediated repression and derepression as well as SO2426-mediated activation of siderophore biosynthesis genes.

\section{Results and Discussion}

\section{Conservation of SO2426 amino acid sequence among} Shewanellae

Previously, we reported that the so2426 gene of S. oneidensis MR-1 shares 27 to $36 \%$ sequence identity at the amino acid level to CpxR and OmpR orthologs from Vibrio cholerae and Escherichia coli [21]. Orthologs of SO2426 were also identified in a number of Shewanella species. Multiple sequence alignment of all available Shewanella SO2426 orthologs revealed a high degree of conservation at key residues (Figure 1). The predicted phosphorylation residues (D18, D19, D62, and K109) associated with the $\mathrm{N}$-terminal CheY-like response regulator domain of SO2426 [21] are highly conserved among Shewanella orthologs. Another striking feature is the high degree of sequence conservation among the Cterminal or output domains of the SO2426 orthologs. This region contains several features of OmpR wingedhelix transcriptional regulators such as the output domain, encompassed by residues T225, G230, and Y231 [22]. Residues 204-215 (LDMHISNTRRKL) resemble the predicted $\alpha 3$-helical region of E. coli CpxR, which comprises the crucial recognition sequence of OmpR-like response regulators [22]. Moreover, it is noteworthy that the annotated 5' terminus of the majority of sequenced Shewanella SO2426 orthologs occurs at M11 relative to the MR-1 sequence (Figure 1). Previous 5' RACE analysis of the transcription start site of MR-1 SO2426 demonstrated that M16 (or M11 relative to the MR-1 sequence) is likely the correct start residue [21].

A phylogenetic tree constructed from the multiple sequence alignment in Figure 1 shows that SO2426 clusters tightly with sequences from Shewanella spp. MR-4, MR-7, and ANA-3 (Figure 2). In a system-wide comparison of Shewanella species, it was recently shown that MR-1, MR-4, MR-7, and ANA-3 tend to be more closely related to each other than to other Shewanellae when comparing genomes, proteomes, gene content, and $16 \mathrm{~S}$ rRNA sequences [23]. These four species exhibit physiological characteristics consistent with their ability to adapt to harsh environments, which is a hallmark characteristic of Shewanella [24]. Strain ANA-3 is most recognized for its ability to respire arsenate [25] but has also been shown to harbor a chromate efflux operon [26], and like MR-1, MR-4 is a known chromate reducer [27]. Synteny of other gene clusters among strains MR-1, MR-4, MR-7, and ANA-3 has been noted for other metabolic processes [28] and cytochrome operons associated with metal reduction [29]. Given the shared genetic and proteomic arrangements among these strains, it is likely that sequence-level relatedness will translate to shared phenotypic traits. 


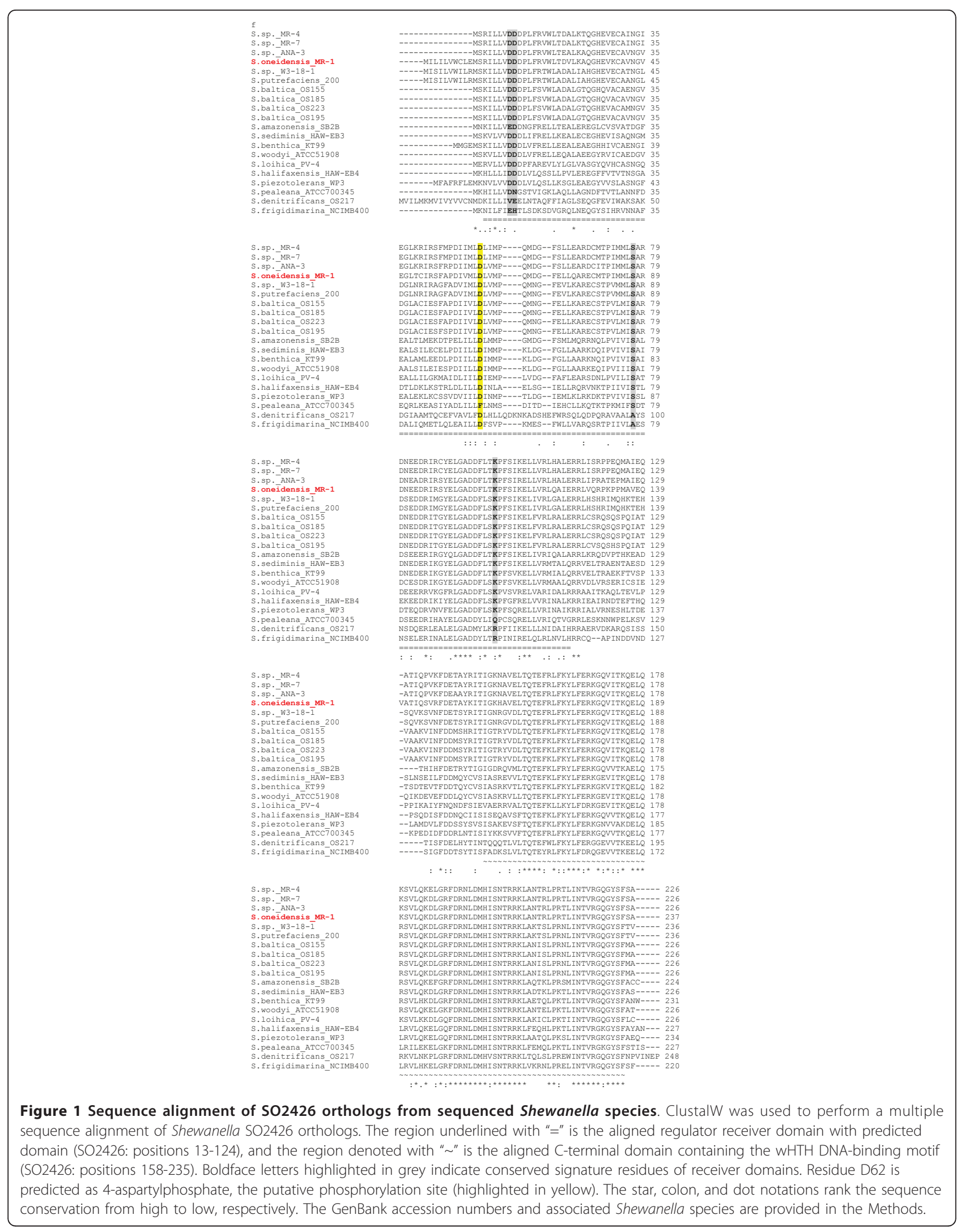




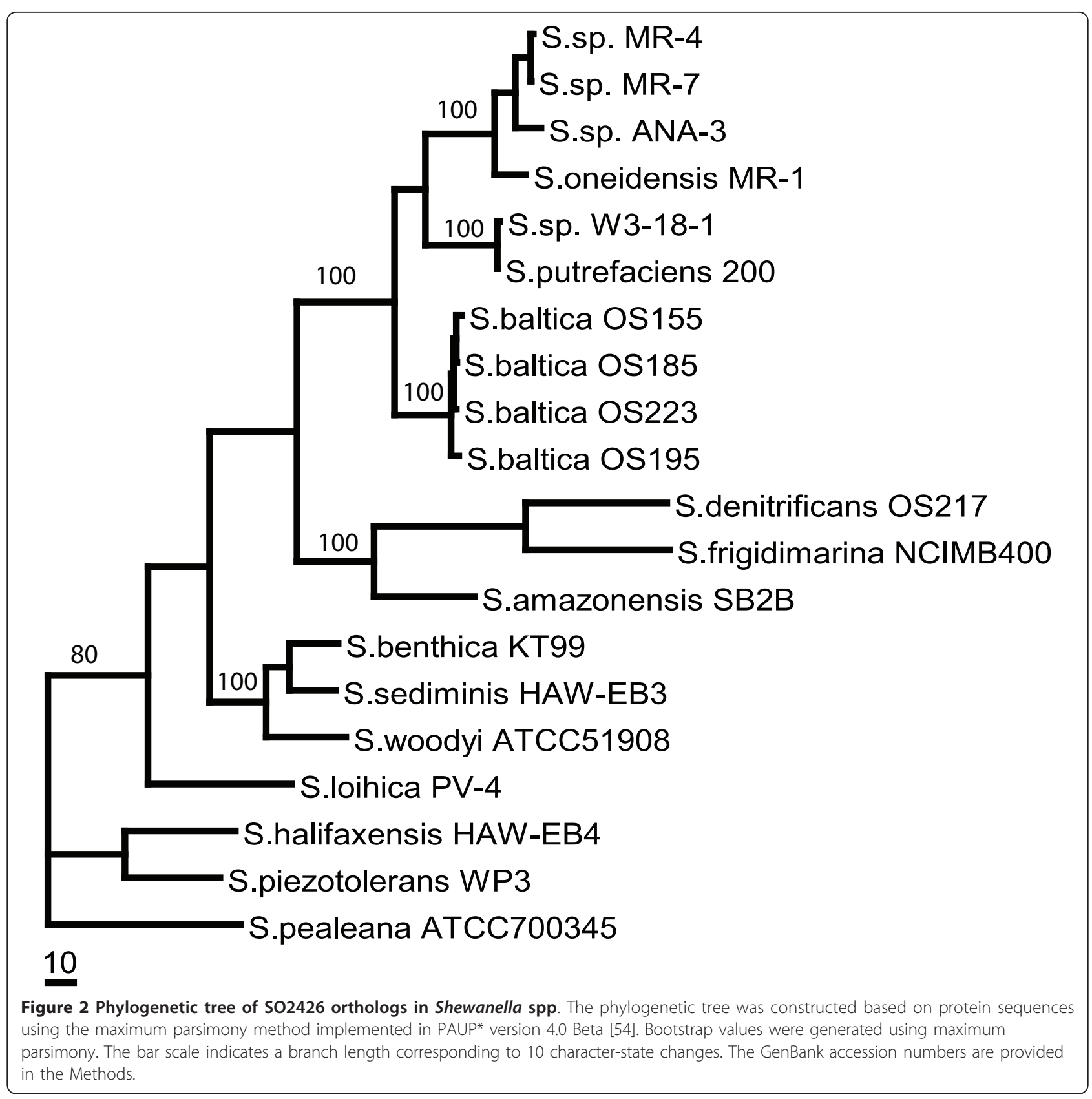

In silico prediction of the SO2426 recognition site

Three complementary computer-based motif search tools-MEME [30], MotifSampler [31], and Gibbs Recursive Sampler [32]-were applied to microarray data generated for a $\Delta s o 2426 \mathrm{MR}-1$ mutant strain [21] in order to predict a consensus recognition site potentially bound by the SO2426 response regulator (see Methods for details). The computational analyses identified a single 14-bp consensus motif in the input dataset (Figure $3)$. This recognition weight matrix consisted of two conserved pentamers (5'-CAAAA-3') in tandem (with the first one being much less conserved), separated by the 4-bp linker sequence 5'-NCAG-3'. The linker sequence composition is not random in that positions 7 and 8 in the motif contain a well-conserved $C$ and $A$ residue, respectively (Figure 3 ). Other two-component response regulators that also recognize a tandem repeat sequence include phosphorylated CpxR (CpxR-P) and OmpR-P. The closest known homolog of $S$. oneidensis SO2426 is CpxR [21]. Intriguingly, the predicted SO2426 recognition sequence resembles the proposed CpxR binding box [5'-GTAAA- $(\mathrm{N})_{5}$-GTAAA-3'] [33,34]. The MR-1 cpxR gene was down-regulated three-fold in $\Delta s o 2426$ mutant cells challenged with chromate [21] compared 


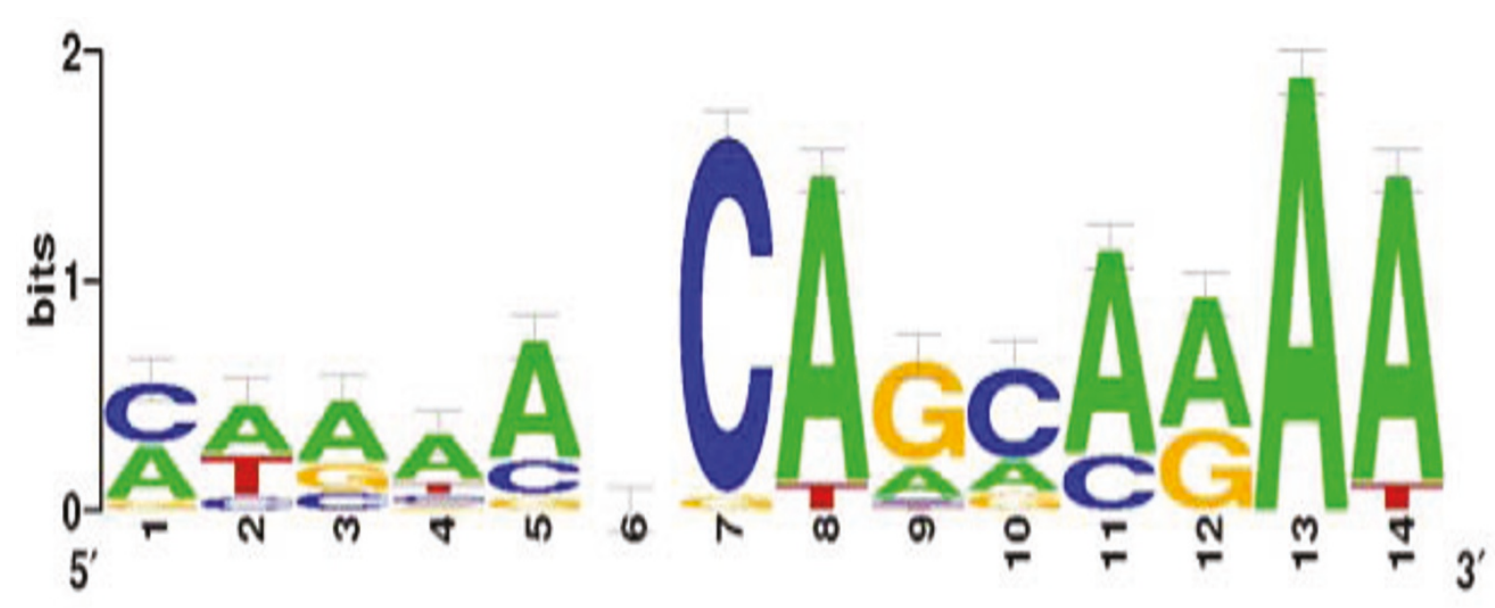

Figure 3 Identification of a predicted consensus SO2426-binding motif in S. oneidensis MR-1 using computational methods. A sequence logo representation [51] of a 14-bp motif model was derived using promoter regions directly upstream of 46 clustered genes exhibiting down-regulated expression in a $\triangle$ so2426 mutant strain of MR-1 [21]. The error bars indicate standard deviations.

to a three-fold induction that was observed for wild-type MR-1 cells under similar conditions [15]. The CpxAR two-component system functions in responding to cell envelope stress and external environmental stimuli, leading to the activation of genes involved in repairing misfolded proteins $[1,35,36]$. The Cpx system has been implicated in a number of cellular responses including the activation of outer membrane porins [37], stationary phase-induced survival mechanisms [38], and $\mathrm{pH}$ stress [39]. Given the activation of CpxR orthologs such as SO2426 during periods of chromate stress in S. oneidensis MR-1 [15,21] and copper stress in E. coli [40], it is suspected that $\mathrm{Cpx}$ and analogous systems operate to overcome oxidative membrane and protein damage induced by exposure to toxic metal ions.

For the present study, we used an input dataset for SO2426 recognition site prediction consisting of 46 genes showing similar down-regulated temporal expression patterns in the $\Delta s o 2426$ mutant [21]. As computational analysis showed, a number of these co-regulated genes were preceded by a conserved tandem repeat (5'-CAAAANCAGCAAAA-3') and included genes $s 02280$ (a putative bcr), so1188, so1190, so3025, so3062, ftn, so1580, so 2045, so3030, so3032, viuA, and so4743 (see Table 1). The majority of these putative SO2426-binding sites were located in upstream regulatory regions, while one site was identified in the so1190 coding region near the annotated 5 terminus at position +12 , suggesting that expression of the so1188-1189-1190 operon might be controlled by internal secondary cis-regulatory elements. In addition, two tandem 5'-CAAAA-3' motifs were identified upstream of the so2426 locus at position - 88 relative to the annotated translation start codon (Table 1), pointing to the possible involvement of an autoregulatory mechanism.
Interestingly, a subset of the genes repressed in the $\Delta$ so2426 mutant, namely genes with functions in iron acquisition and storage, also possessed a predicted ferric uptake regulator (Fur) box in their upstream regulatory regions. A potential Fur recognition motif, 5'-AAATGAtATTGATTcTCgTTT-3', was identified in the upstream region flanking so2426 and overlapped the transcriptional start sites for this gene [21].

Several lines of evidence further support the role of so2426 in the regulation of iron acquisition in S. oneidensis MR-1. A recent study applying gene network reconstruction to MR-1 indicated that SO2426 clusters with iron acquisition genes in a distinct iron-responsive network system [14]. Within this iron acquisition gene network were a number of members of the SO2426 regulon proposed here, namely, so1188, so1190, so3025, so3030-30313032, so3063, and so4743 [14]. All of these genes, including so2426, were up-regulated under iron-depleted growth conditions compared to iron-replete conditions. Additionally, so3030 was up-regulated almost 14-fold in a fur mutant, while genes so3031-so3033 were up-regulated 4 to 11-fold [13]. A separate transcriptomic study with a fur deletion mutant revealed that the gene with the greatest expression change in the fur mutant compared to the MR1 wild-type strain was so2426, which showed a 30- and 26fold increase in expression at the transcript level under aerobic and anaerobic growth conditions, respectively [12]. In addition to the enhanced expression of so2426 in a fur mutant, this gene has been shown to be up-regulated under chromate [15,41] and strontium [42] stress.

The presence of a putative Fur-binding sequence in the promoter region of so2426 suggests that expression of this response regulator may be subject to multiple levels of regulatory control. Identification of a Fur box 
Table 1 Putative SO2426 gene targets containing the predicted SO2426-binding site

\begin{tabular}{|c|c|c|c|c|c|}
\hline ORF & Functional Category/Gene Product & Motif & Strand & Distance $^{a}$ & $E_{\text {-value }}^{b}$ \\
\hline & Cellular processes & & & & \\
\hline \multirow[t]{3}{*}{ SO2280 } & bicyclomycin resistance protein & AACGCTCAGGCAAA & - & -241 & 2.06E-04 \\
\hline & Central intermediary metabolism & & & & \\
\hline & 5-methylthioadenosine nucleosidase/S- & & & & \\
\hline \multirow[t]{2}{*}{ SO3705 } & adenosylhomocysteine nucleosidase, putative & GTCAGCCAGCAAAA & + & +21 & 4.73E-05 \\
\hline & Energy metabolism & & & & \\
\hline \multirow[t]{2}{*}{ SO2743 } & acetyl-coenzyme A synthetase (acs) & AAAAAAGAGCAAAA & - & -160 & 1.46E-05 \\
\hline & Hypothetical proteins & & & & \\
\hline SO1188 & conserved hypothetical protein & AAAACTCAGCAGAA & - & -113 & 2.08E-06 \\
\hline SO1190 & conserved hypothetical protein & CTAAGGCAACAAAA & - & +12 & $2.38 \mathrm{E}-05$ \\
\hline SO1770 & glycerate kinase, putative & ACAACCCAGAAGAA & - & -177 & 2.61E-05 \\
\hline SO3025 & conserved hypothetical protein & GCAAAACATCAAAA & + & -234 & 1.13E-04 \\
\hline SO3062 & hypothetical protein & ATAAATCAGGAGAA & + & -5 & 7.64E-06 \\
\hline SO4499 & hypothetical protein & CTGCAACAGGAGAA & + & -5 & 1.19E-05 \\
\hline SO4504 & conserved hypothetical protein & ATGTCCCAGACAAA & + & -169 & 1.06E-04 \\
\hline \multirow[t]{2}{*}{ SO4719 } & conserved hypothetical protein & ATGAACCACAAGAA & + & -199 & $9.88 \mathrm{E}-05$ \\
\hline & Transport and binding proteins & & & & \\
\hline SO0139 & ferritin (ftn) & CAAAAGCAACAAAA & - & -63 & $2.08 \mathrm{E}-06$ \\
\hline SO1580 & TonB-dependent heme receptor & AAAAAGCAGAAAAA & - & -112 & $3.68 \mathrm{E}-06$ \\
\hline SO1771 & permease, GntP family & CTACAACAGCCAAA & + & -41 & 2.81E-06 \\
\hline SO2045 & cation efflux family protein & CACCCTCAACAGAA & + & +11 & 5.98E-05 \\
\hline SO3030 & siderophore biosynthesis protein $(a / c A)$ & CTGTAACAGCAAAT & + & -133 & 2.86E-05 \\
\hline SO3032 & siderophore biosynthesis protein, putative & CCGGATCAGCAAAA & + & -284 & 1.46E-05 \\
\hline SO3033 & ferric alcaligin siderophore receptor & ATCAAACAGCCAAA & + & -112 & $3.20 \mathrm{E}-06$ \\
\hline SO3063 & sodium:alanine symporter family protein & CAAAAACAACAGAA & + & -18 & 1.09E-06 \\
\hline SO4150 & transporter, putative & AAAAAACTGCAGAA & + & +16 & 7.64E-06 \\
\hline SO4516 & ferric vibriobactin receptor (viuA) & CAGTAGCAGAAGAA & + & -249 & $1.62 \mathrm{E}-05$ \\
\hline \multirow[t]{2}{*}{ SO4743 } & TonB-dependent receptor, putative & CAAAAACAACAAAT & - & -168 & $2.38 \mathrm{E}-05$ \\
\hline & Signal transduction & & & & \\
\hline SO2426 & DNA-binding response regulator & CAATACCTGCCAAA & + & -88 & $5.12 \mathrm{E}-05$ \\
\hline
\end{tabular}

${ }^{a}$ Distance in base pairs of the start of the potential SO2426 binding site from the first nucleotide of the predicted translation start codon of the corresponding gene listed in the first column. ${ }^{b}$ The E-value of the motif is an estimate of the number of motifs with the same width and number of occurrences that would have equal or higher log likelihood ratio if the training set sequences had been generated randomly according to the background model [30].

immediately downstream of the -10 promoter element and up-regulation of so2426 expression in a fur deletion mutant are both consistent with repression of this gene by Fur under iron-sufficient conditions. Similarly, of those genes encoding transport and binding proteins, ftn, so1580, the so3030-3031-3032 operon, so4516, and so4743 are probable members of the Fur regulon based on their derepressed expression patterns in a S. oneidensis $\Delta$ fur mutant and the presence of a putative Fur box in their respective upstream regions [12]. Collectively, these observations suggest cross-regulation of ironresponsive and other metal-responsive gene networks in S. oneidensis MR-1.

\section{SO2426 binds in region of predicted recognition site upstream of alcA}

Given the potential overlap in the response of S. oneidensis to iron and other metals, we chose to focus on the
S. oneidensis siderophore biosynthesis operon in testing the interaction of two recombinant versions of the $\mathrm{SO} 2426$ protein with its predicted binding motif. The direct involvement of so3030-3031-3032 in the production of hydroxamate-type siderophores was recently demonstrated with deletion mutants within this gene cluster [43]. Induction of the so3030-3031-3032 operon in MR-1 cells under chromate challenge compared to unperturbed cells has been demonstrated using both transcriptomic and proteomic approaches $[15,16]$. In further studies with a so2426 deletion mutant under chromate challenge, the so3030-3031-3032 operon was significantly down-regulated $[21,41]$. These data, together with the predicted SO2426-binding motif upstream of so3030, suggest that SO2426 directly regulates siderophore production in strain MR-1 under conditions of chromate stress. We employed electrophoretic mobility shift assay (EMSA) to determine if the SO2426 protein was able to interact with the 
predicted binding sequence upstream of the so3030-30313032 operon.

Our previous 5' RACE studies demonstrated that the likely $5^{\prime}$ terminus of SO2426 occurs at a methionine located at position 11 downstream from the originally annotated translation start [21]. Comparative sequence analysis of SO2426 with the CpxR and OmpR amino acid sequences from $V$. cholerae and $E$. coli showed that sequence homology between conserved receiver domains for these other well-characterized response regulators and SO2426 begins 13 amino acids downstream of the annotated start site for SO2426 [21]. This conservation is further observed for the Shewanella SO2426 orthologs (Figure 1). In order to test the functionality of the shorter version of SO2426, both the full-length annotated form (designated SO2426) and the "short" form beginning with M11 (designated SO2426sh), were expressed using the pTrcHis expression vector system, which incorporates an $\mathrm{N}$-terminal six-histidine tag for affinity purification. The His-tagged proteins were expressed in E. coli and partially purified from crude cell extracts by Ni-affinity column purification (see Methods for details). Expression of the recombinant SO2426 protein was determined by SDS-PAGE (Figure 4A) and Western blotting (Figure 4B), which confirmed the presence of this protein within the expected size range of 26-27.4 $\mathrm{kDa}$. Similar SDS-PAGE and immunoblotting results were obtained for the verification of recombinant SO2426sh expression (data not shown).

A digoxigenin-labeled DNA probe spanning the predicted SO2426-binding site motif upstream of the so30303031-3032 operon (Figure 5, double underlined region), but excluding the putative Fur box, was generated by PCR amplification and used as the DNA probe in measuring the DNA-binding activity of the partially purified recombinant SO2426 and SO2426sh proteins. Figure 6A shows that the DNA probe shifted upward in the presence of recombinant SO2426, with the shift becoming incrementally more enhanced as the protein concentration in the EMSA reaction mixture was increased. No shift was observed with protein extracts prepared from $\mathrm{pTrcHis}$ vector-only $E$. coli control strains, ruling out the possibility that the probe shift was due to non-specific binding of contaminating proteins. A comparable shift was observed for recombinant SO2426sh (Figure 6B), thus supporting our proposition that the actual 5 ' terminus of the SO2425 occurs at residue M11. Gel shift assays performed with additional DNA probes upstream of the so3030-3031-3032 operon as well as so3036, which also contains a putative SO2426 recognition sequence, showed a band-shift in the presence of recombinant SO2426 (data not shown). Although the primary focus in this study is the functional role of SO2426 in siderophore production, future studies will be necessary to analyze the interaction of SO2426 with additional recognition sites to further define its regulon.

\section{Siderophore production is deficient in a $\Delta$ so2426 mutant strain}

Earlier physiological evidence for the role of SO2426 in siderophore production was obtained using liquid CAS assays in which relative siderophore production levels for the $\Delta s o 2426$ mutant were compared to those for the wild-type MR-1 strain [21]. These studies demonstrated that the deletion mutant was markedly deficient in siderophore synthesis compared to the wild-type strain in LB medium supplemented with chromate [21]. LB
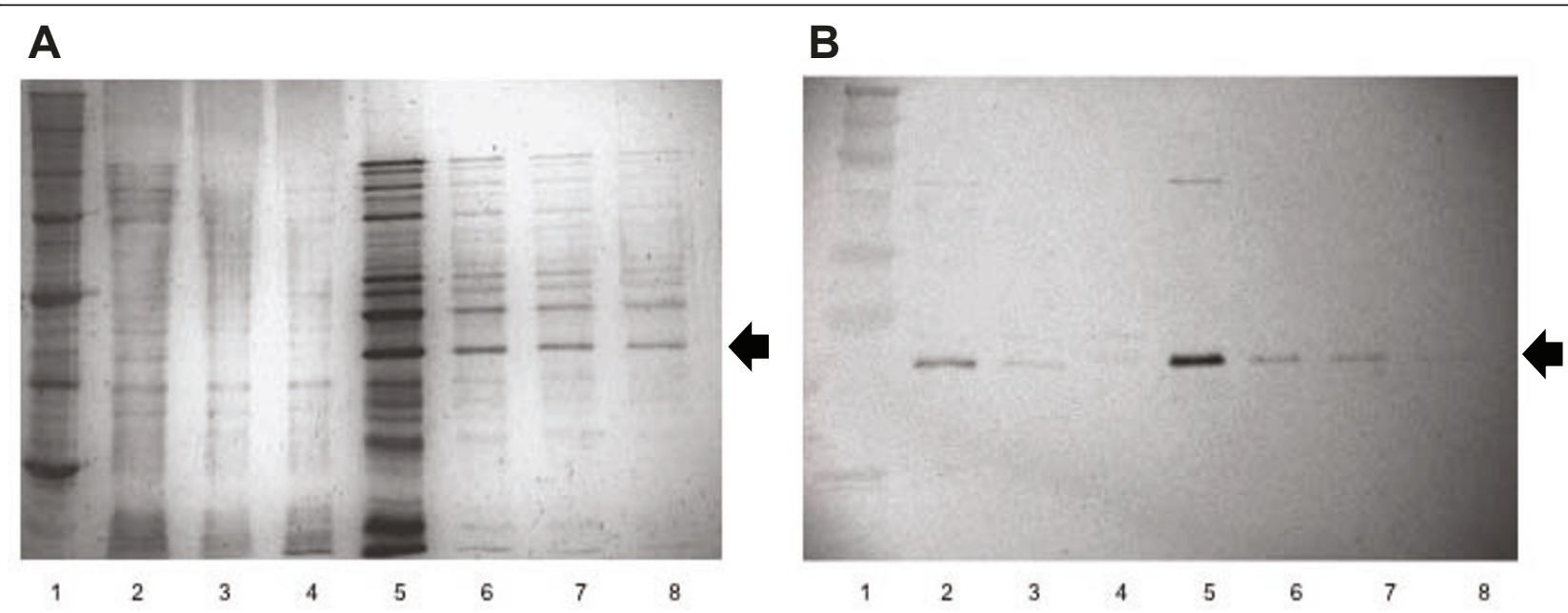

Figure 4 Partial purification (A) and Western blot (B) verification of recombinant SO2426 protein. Panel A, silver-stained gel of partial purification using a Ni-affinity column. Panel B, Western blot analysis performed in parallel with Anti-HisG Antibody (Invitrogen). Lanes: 1, MW markers; 2, cell lysate; 3, Wash 1; 4, Wash 2; 5-8, Elution Fractions 1-4. Recombinant SO2426 is denoted with an arrow. 
... TССтTATTCCCAATTTATCAACTCACCGACAAACCGTATTTCAGCTCCTTATTTA

FUR BOX

CCAGTCAAAAGTACGACTCATCCGACAACCTGCAGATGAGAACGATTTGCATTTATAAT

so2426 Motif

TTTTAAGATGCTAATTA TTCGTCGTTGTAGTGAAC TGTAACAGCAAAT CAGACGGAA

AAATTTCTATTTAGCAACACAATGGAATGACTTACACGGCAGCTGCAGAGCGATCAACT

СTTCTACTCATCCATCAGTCGATGGTGGCAGCAACCGTTTTAGCAGGAATTTAAGGAGG

CGTAAGTGACTACCCCAAAAAAGGAAATCGATCGTACGATTTACGATCTACTCGGCATT

GGCATAGGTCCATTCAATCTAGGACTGGCGGCACTGTGTGAACCTATTAATGACTTAAG

CTGTTTGTTTTTAGATGCAAAAACCGAGTTTGATTGGCACCCTGGTATGCTGATC . . .

Figure 5 Upstream nucleotide sequence of the siderophore biosynthesis so3030-3031-3032 operon. The recognition site (Fur Box) for the ferric uptake regulator (single underline) and the predicted SO2426-binding motif (red type) are noted in the upstream region. A DNA probe for EMSA studies flanking the SO2426-binding motif was generated by PCR amplification (double underlined sequence). The $5^{\prime}$ coding region of so3030 is highlighted in salmon.

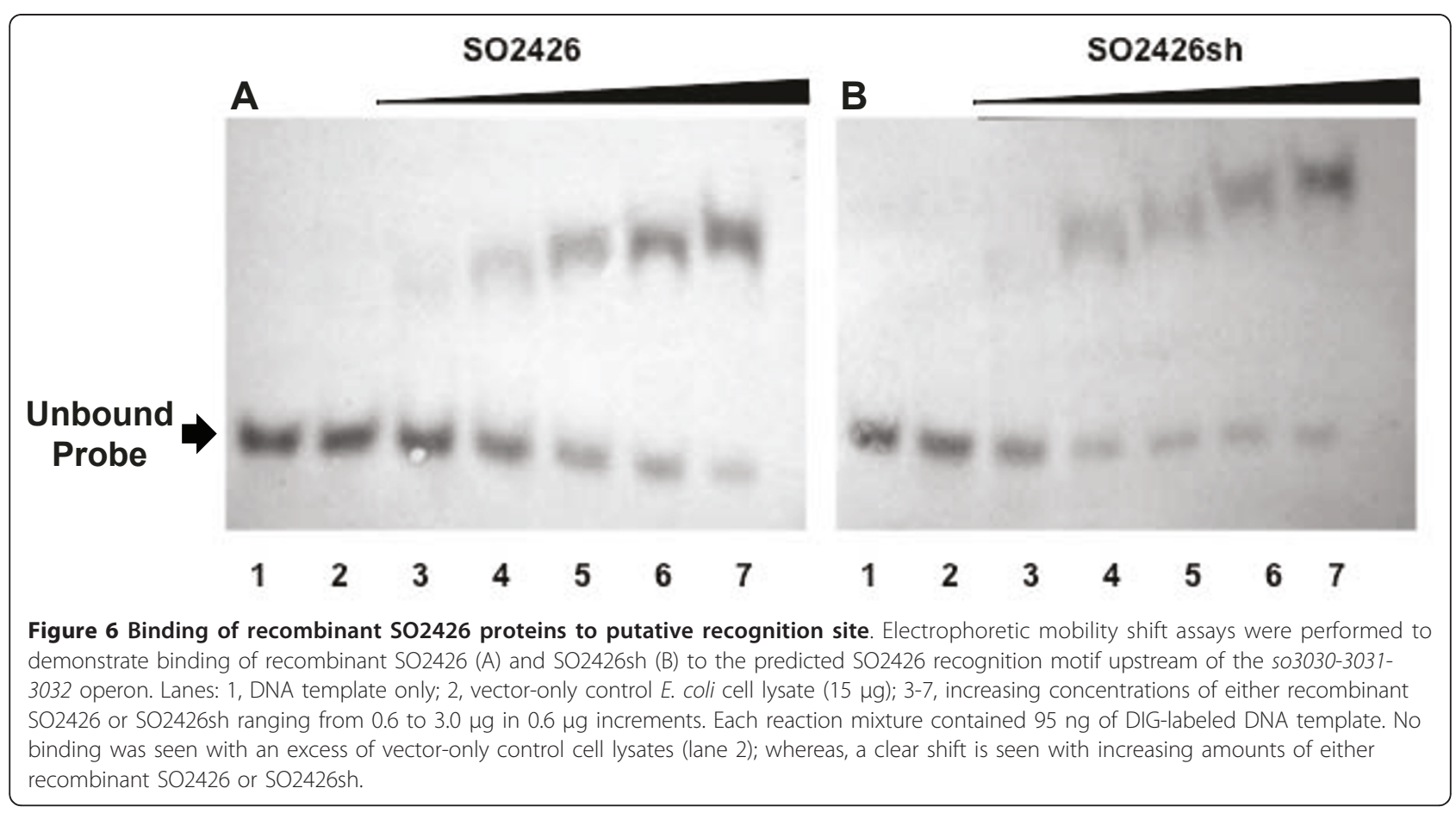


medium constitutes a sufficient source of iron $(\sim 17 \mu \mathrm{M})$ [13]. Additionally, under iron-replete conditions, in which $50 \mu \mathrm{m} \mathrm{FeCl}$ was added to the medium, there was no change in siderophore levels in the $\Delta$ so2426 mutant. Conversely, siderophore production in the wildtype MR-1 strain returned to background levels in the presence of added iron [21].

We expanded upon our previous physiological work by examining siderophore production under artificially imposed conditions of iron depletion. To achieve this, wild-type MR-1 and $\Delta s o 2426$ mutant strains were allowed to grow in LB medium supplemented with $80 \mu \mathrm{M}$ of the Fe chelator 2,2'-dipyridyl to simulate iron-limiting conditions. Other studies demonstrated that a 2,2'-dipyridyl concentration of $\leq 100 \mu \mathrm{M}$ had a negligible effect, if any, on the growth rate of $S$. oneidensis MR-1 and certain mutant strains under aerobic conditions [14,43]. Similarly, we observed that MR-1 and the $\Delta$ so2426 mutant could grow aerobically at relatively normal rates in LB supplemented with $80 \mu \mathrm{M}$ of 2,2'-dipyridyl (Figure 7A), indicating that environmental Fe was not scavenged below a critical Fe threshold necessary for growth. As shown in Figure 7B, the $\Delta s o 2426$ mutant was unable to produce CAS-reactive siderophores at wild-type rates under aerobic growth conditions in the absence of 2,2'-dipyridyl. This deficiency was enhanced in the presence of iron chelator (Figure 7B).
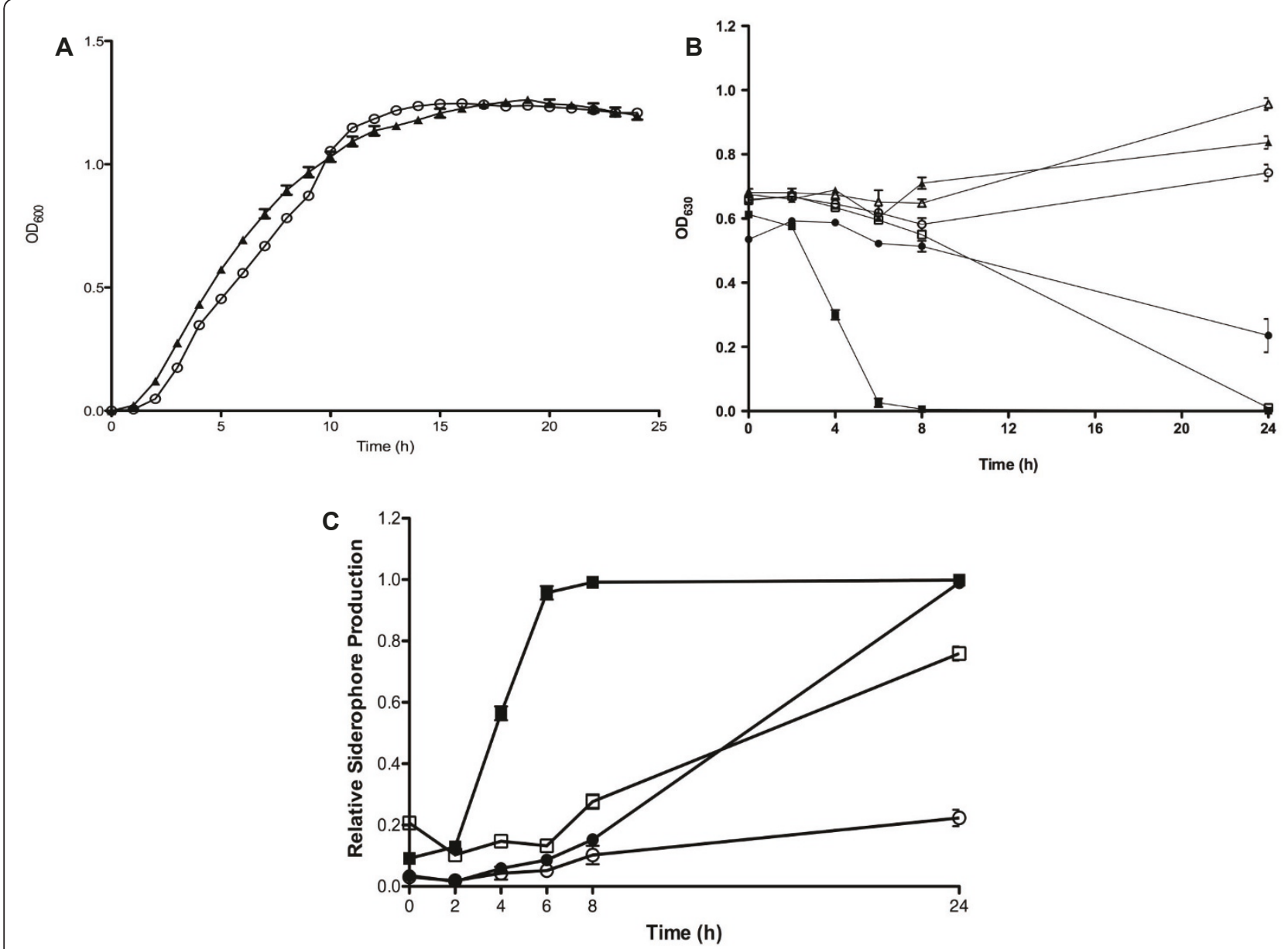

Figure 7 Growth capacity and siderophore production by wild-type MR-1 and $\Delta$ so2426 strains in the presence of 2,2'-dipyridyl. (A) Aerobic growth of wild-type MR-1 (closed triangles) and the $\triangle$ so2426 mutant (open circles) in LB supplemented with $80 \mu \mathrm{M}$ of the Fe chelator 2,2'-dipyridyl. Cell growth was assessed for triplicate cultures and plotted as the mean $\mathrm{OD}_{600} \pm \mathrm{SEM}$. (B) Absorbance at $630 \mathrm{~nm}$ of CAS-treated samples in the absence (open symbols) and presence (closed symbols) of 2,2'-dipyridyl. Results are shown for wild-type MR-1 (squares), the $\triangle$ so2426 mutant (circles), and LB only (triangles). (C) Relative production of CAS-reactive siderophores by wild-type MR-1 (closed symbols) and the $\triangle$ so2426 mutant (open symbols) under aerobic growth conditions. 2,2'-dipyridyl $(80 \mu \mathrm{M})$ was added to mid-log-phase $\left(\mathrm{OD}_{600}, 0.6\right)$ MR-1 and $\triangle$ so2426 mutant cultures cultivated in LB broth, and relative siderophore synthesis was monitored over time using the CAS-based siderophore detection assay. The relative siderophore production was calculated by subtracting the supernatant $A_{630}$ (absorbance at $630 \mathrm{~nm}$ ) for the wild type or mutant from the control (uninoculated LB medium) and then determining the ratio of corrected supernatant $A_{630}$ to control $A_{630}$. Error bars represent the standard error of the mean for three replicate CAS measurements. Circles represent unamended LB cultures; squares represent iron-depleted cultures. 
Relative siderophore production by wild-type MR-1 increased sharply, attaining a maximum level at the 6-h time point following exposure to 2,2'-dipyridyl (Figure 7C). At this time interval, we detected an 11-fold increase in the synthesis of CAS-reactive siderophores for MR-1 under iron depletion compared to MR-1 under iron-sufficient conditions (LB only). In the same 6-h time period, there was only a marginal elevation in siderophore production by the $\Delta s o 2426$ mutant, which exhibited substantially reduced levels of siderophore production compared to MR-1 under iron depletion conditions (Figure 7C).

The impaired ability of the $\Delta s o 2426$ mutant to produce siderophores during aerobic growth suggests that so2426 is a positive regulator of siderophore production in S. oneidensis MR-1. As noted earlier, several of the genes predicted to belong to the so2426 regulon also have Fur-binding motifs in their upstream regions. The likely molecular mechanism controlling iron homeostasis in $S$. oneidensis MR-1 involves Fur-mediated transcriptional repression, which includes down-regulation of so2426 expression under iron-replete conditions and derepression followed by SO2426-mediated transcriptional activation under iron-limited conditions. This may explain the residual siderophore production in the $\Delta s o 2426$ mutant. It is also possible that an as-yet uncharacterized secondary mechanism for siderophore production exists in strain MR-1.

\section{Conclusions}

SO2426 is annotated as a DNA-binding response regulator, but its specific function in S. oneidensis MR-1 was previously undefined. Using combined in silico motif prediction and in vitro binding assays along with physiological characterization, this report provides an important empirical step toward describing the $\mathrm{SO} 2426$ regulon. We initially identified a putative SO2426-binding consensus motif that consists of two conserved pentamers (5'CAAAA-3') in tandem. Electrophoretic mobility shift assays demonstrated that recombinant $\mathrm{SO} 2426$ exhibits binding specificity with its predicted motif within the 5 , regulatory region flanking a siderophore biosynthesis operon. A $\Delta s o 2426$ mutant was unable to synthesize CASreactive siderophores at wild-type rates under iron limitation. Collectively, these data support a function for SO2426 as a positive regulator of siderophore-mediated iron acquisition in S. oneidensis MR-1.

In addition to exhibiting iron-responsive expression, the so2426 gene has been previously shown to be up-regulated in response to chromate stress [15,41]. The up-regulation of iron acquisition and iron storage systems in response to metal stress is not unique to S. oneidensis. In Arthrobacter sp. FB24, a number of proteins with putative functions in iron sequestration, such as Ferritin-Dps family proteins, as well as Reiske (2Fe-2S) domain proteins, showed increased abundance as a result of chromate stress [17]. Copper has been shown to disrupt Fe-S clusters in important enzymes in E. coli [44]. An E. coli strain defective in iron transport was also found to be more sensitive to chromium [19]. Exposure to manganese in B. subtilis resulted in altered intracellular iron pools with subsequent expression of Furregulated genes [45]. The reason for the up-regulation of iron-responsive genes is unclear. It has been speculated that metal ions such as chromate result in oxidative stress mediated through Fenton-type reactions with ferrous iron [18,46-48]. Up-regulation of iron storage proteins may help alleviate metal-induced oxidative damage by binding excess $\mathrm{Fe}$ and preventing its interaction with other metal ions. It is also apparent that proteins with $\mathrm{Fe}-\mathrm{S}$ prosthetic groups as part of their active centers are primary targets of metalinduced damage. These processes undoubtedly disrupt intracellular iron homeostasis, leading to the up-regulation of iron acquisition and sequestration systems. The evidence provided here and in our previous work strongly points to an integral role of SO2426 in such iron control systems.

\section{Methods}

\section{Bacterial strains, plasmids, and culture conditions}

All strains and plasmids used in this study are described in Table 2. E. coli strains were cultured aerobically in LuriaBertani (LB) [Difco, Detroit, MI] medium at $37^{\circ} \mathrm{C}$ with shaking. For recombinant $E$. coli strains, ampicillin was added to $\mathrm{LB}$ at a concentration of $50 \mu \mathrm{g} / \mathrm{ml}$. S. oneidensis strains were grown aerobically in $\mathrm{LB}$ medium at $30^{\circ} \mathrm{C}$ with shaking at 200 RPM.

\section{SO2426 weight matrix development and identification of a putative SO2426 recognition site}

MEME [30], MotifSampler [31], and Gibbs Recursive Sampler [32] were used to predict promoter recognition sequences potentially bound by SO2426. To facilitate motif searching, the time-series microarray expression profiles of the $\Delta s o 2426$ relative to the parental strain were clustered using Hierarchical Clustering Explorer (HCE) [49]. During the clustering process, only genes with an expression value of at least $\geq 2$-fold or $\leq 0.5$-fold in one or more of 6 expression profiling time points were included in the analyses. As a result, a dataset of 841 genes was clustered based on the average linkage using Euclidean distance [21]. We extracted a sub-cluster comprising 46 similarly down-regulated genes throughout the 6 time points, and this dataset was used as the input data for putative SO2426 bindingsite prediction. The consensus SO2426-binding sequence was predicted with MEME using the following parameters: (i) the motif width ranged from 6 to 50; (ii) the total number of sites in the training set where a single motif occurred was 3; and (iii) the sequence had 0 or 1 binding site. MAST [50] was used to scan the sequence database with the predicted MEME-derived motif. The Gibbs Recursive Sampler 
Table 2 Bacterial strains and plasmids used in this study

\begin{tabular}{|c|c|c|}
\hline Bacterial Strains & Genotype & Source/Reference \\
\hline Shewanella oneidensis MR-1 & Wild type & ATCC 7005500 Lab stock \\
\hline $\mathrm{MR}-1 / \Delta \mathrm{so} 2426$ & Deletion of so2426 locus & {$[21]$} \\
\hline E. coli TOP10 & Cloning and expression strain & Invitrogen \\
\hline E. coli ER2508 & Major proteinase-deficient strain & New England Biolabs \\
\hline His-ER-2426-1-1 & Expresses full-length $\mathrm{SO} 2426$ protein & This study \\
\hline His-Top-26s-4 & Expresses truncated SO2426 protein & This study \\
\hline E. coli (pTOPO) & Vector-only control & Invitrogen \\
\hline \multicolumn{3}{|l|}{ Plasmids } \\
\hline pTrcHis-2426sh & so2426sh cloned in frame with N-terminal polyhistidine & This study \\
\hline pTrcHis-2426 & so2426 cloned in frame with $\mathrm{N}$-terminal polyhistidine & This study \\
\hline
\end{tabular}

program was performed as described previously [12]. MotifSampler [31] was employed to confirm the consensus motif predicted using MEME and Gibbs Recursive Sampler. A sequence logo [51] was generated to graphically represent the sequence conservation of the predicted SO2426 recognition site.

\section{Sequence analysis of SO2426 orthologs}

ClustalW [52] was used to perform a multiple sequence alignment of Shewanella SO2426 orthologs. Conserved signature residues in the receiver domain of response regulators were annotated based on reference [53]. The phylogenetic tree was constructed based on protein sequences using maximum parsimony method implemented in PAUP* version 4.0 Beta [54]. The bootstrap values were generated using maximum parsimony. The GenBank accession numbers are as follows: YP_734035.1, Shewanella sp. MR-4; YP_738119.1Shewanella sp. MR-7; YP_750834.1, Shewanella frigidimarina NCIMB 400; YP_869596.1, Shewanella sp. ANA-3; YP_927593.1, Shewanella amazonensis SB2B; YP_963447.1, Shewanella sp. W3-18-1; ZP_01705802.1, Shewanella putrefaciens 200; YP_001050420.1, Shewanella baltica OS155; YP_001094061.1, Shewanella loihica PV-4; YP_001366502.1, Shewanella baltica OS185; YP_001474053.1, Shewanella sediminis HAWEB3; YP_001502091.1, Shewanella pealeana ATCC 700345; YP_001554844.1, Shewanella baltica OS195; ZP_02156174.1, Shewanella benthica KT99; YP_001674438.1, Shewanella halifaxensis HAW-EB4; YP_001760668.1, Shewanella woodyi ATCC 51908; YP_002311920.1, Shewanella piezotolerans WP3; YP_002357973.1, Shewanella baltica OS223; NP_718016.1, Shewanella oneidensis MR-1; and YP_562912.1, Shewanella denitrificans OS217.

\section{Siderophore detection}

The chrome azurol-S (CAS)-based assay for detection of siderophore production during cellular growth in liquid was performed as described elsewhere $[21,55]$ with slight modifications in culture conditions. Overnight LB cultures of the $\Delta$ so2426 strain and the wild-type MR-1 strain were used to inoculate fresh LB broth and allowed to grow to mid-logarithmic phase $\left(\mathrm{OD}_{600} \sim 0.6\right)$. The midlog-phase cultures were amended with $50 \mu \mathrm{M} \mathrm{FeCl}_{3}, 80$ $\mu \mathrm{M} 2,2$ '-dipyridyl, or $0.3 \mathrm{mM} \mathrm{K}_{2} \mathrm{CrO}_{4}$. A control consisting of LB without amendment was prepared for each strain. The cultures were allowed to incubate for $24 \mathrm{~h}$ at $30^{\circ} \mathrm{C}$ with shaking. Aliquots were taken for CAS assay analysis at $0,2,4,6,8$, and $24 \mathrm{~h}$ post amendment. Cellfree supernatants were mixed 1:1 with the CAS assay solution and equilibrated at room temperature for $2 \mathrm{~h}$ prior to reading the absorbance at $630 \mathrm{~nm}$. The relative production of CAS-reactive siderophores was calculated as described [21] and reported as the average of three independent experiments.

\section{Expression and partial purification of recombinant SO2426 protein}

Bacterial expression vectors were constructed by cloning the full-length SO2426 gene and a shortened form (SO2426sh) in frame with the N-terminal His-tag of pTrcHis (Invitrogen, Carlsbad, CA). Plasmids were transformed into E. coli TOP10 (Invitrogen, Carlsbad, CA) or E. coli ER2508 (New England Biolabs, Ipswich, MA) host cells. Transformants were selected on LBampicillin agar plates. Positive clones were verified by sequence analysis at the Purdue Genomics Core Facility.

Cells carrying the expression vectors were grown at $37^{\circ} \mathrm{C}$ in $100 \mathrm{ml}$ of LB with $50 \mu \mathrm{g} / \mathrm{ml}$ ampicillin until a cell density of $\sim 0.6$ was attained. IPTG was added to a concentration of $1 \mathrm{mM}$, and the cultures were incubated for an additional 3 hours to induce expression of recombinant SO2426 proteins. Cells were harvested by centrifugation and washed in $1 \mathrm{X}$ TBS. Cell lysates were prepared by sonicating cell pellets in Guanidium Lysis Buffer, pH 7.8 (Invitrogen, Carlsbad, CA) containing 1X Complete-Mini Protease Inhibitor Cocktail (Roche Applied Science, Indianapolis, IN). The lysates were centrifuged at 6,000 RPM for $10 \mathrm{~min}$ to remove cell debris. 
His-tagged proteins were recovered from cell lysates using the ProBond Purification System (Invitrogen, Carlsbad, CA) under hybrid conditions as specified by the manufacturer's protocol. A total of eight 1 to $2-\mathrm{ml}$ elution fractions were collected for each protein extract.

\section{Verification of SO2426 recombinant protein}

Expression of His-tagged SO2426 and SO2426sh proteins in the elution fractions was verified by Western blot analysis using the Western Breeze Chromogenic Western Blot Immunodetection Kit (Invitrogen, Carlsbad, CA). Histagged proteins were probed with an anti-HisG antibody (Invitrogen, Carlsbad, CA) with secondary detection using anti-mouse IgG-alkaline phosphatase antibody provided in the Western Breeze kit. Positive elution fractions were pooled and concentrated with YM-3 Centricon Centrifugal Filter Devices (Millipore, Billerica, MA). Concentrated fractions were dialyzed overnight at $4{ }^{\circ} \mathrm{C}$ against TED buffer [20 mM Tris-Cl (pH 7.0), $150 \mathrm{mM} \mathrm{NaCl}, 0.1 \mathrm{mM}$ EDTA, and $0.1 \mathrm{mM}$ DTT] using mini dialysis tubes with a molecular weight cutoff of $8 \mathrm{kDa}$. Protein concentration was determined using a Nanodrop ND-1000 Spectrophotometer (Rockland, DE).

\section{Electrophoretic Mobility Shift Assay (EMSA)}

A non-labeled DNA probe was first generated by PCR amplification of an 83-bp region upstream of so3030 using primers klh001 and klh004 (Table 3) and S. oneidensis MR-1 genomic DNA as a template. The probe sequence was verified by sequence analysis at the Purdue Genomics Core Facility. This PCR product was then used as the template in a PCR amplification reaction to generate a Digoxigenin-labeled DNA probe for use in EMSA. The reaction mixture consisted of $25 \mathrm{mM} \mathrm{MgCl}_{2}$, $1 \mathrm{X}$ Promega Go-Flexi Taq Polymerase buffer, a 1:6 ratio of dTTP:DIG-11-dUTP dNTP mix, $0.2 \mathrm{mM}$ each of primers klh001 and klh004, $5.5 \mathrm{ng}$ of the unlabeled PCR product as a template, and $10 \mathrm{U}$ of Taq to $1 \mathrm{U}$ Pfu cocktail in a final reaction volume of $50 \mu \mathrm{l}$. The PCR amplification cycle consisted of $95^{\circ} \mathrm{C}$ for $4 \mathrm{~min}$ and 30 cycles of $94^{\circ} \mathrm{C}$ for $1 \mathrm{~min}, 50^{\circ} \mathrm{C}$ for $30 \mathrm{sec}$, $72^{\circ} \mathrm{C}$ for $1 \mathrm{~min}$, with a final extension step at $72^{\circ} \mathrm{C}$ for $5 \mathrm{~min}$. Labelling efficiency was verified by Southern blot analysis using the DIG Nucleic Acid Detection Kit (Roche Applied Science, Indianapolis, IN) according to the

\section{Table 3 Oligonucleotide primers used in this study}

\begin{tabular}{lll}
\hline Primer & DNA sequence $\left(\mathbf{5}^{\prime} \boldsymbol{\rightarrow} \mathbf{3}^{\prime}\right)$ & Reference or source \\
\hline klh001 & TTCGTCGTTGTAGTGAACC & This study \\
klh004 & TGCCGTGTAAGTCATTCC & This study \\
$2426 F$ & ATGATATTGATTCTCGTTGGT & This study \\
$2426 \mathrm{R}$ & TTAAGCGCTAAAACTGTATCCTTG & This study \\
$2426 \mathrm{shF}$ & ATGAGTAGAATACTGTTAGTCGAT & This study \\
$2426 \mathrm{shR}$ & TTAAGCGCTAAAACTGTATCC & This study \\
\hline
\end{tabular}

manufacturer's protocol for colorimetric detection. The DIG-labeled DNA probe was quantified using a Nanodrop ND-1000 Spectrophotometer.

EMSA was performed in 20 - $\mu$ l reaction volumes containing 0.5X EMSA buffer [5 mM Tris- $\mathrm{Cl}$ ( $\mathrm{pH} 8.0)$, $75 \mathrm{mM} \mathrm{KCl}, 0.05 \mathrm{mM}$ DTT, $0.05 \mathrm{mM}$ EDTA, 6\% glycerol], $5 \mathrm{mM} \mathrm{MgCl} 2,20 \mathrm{mM}$ Acetyl- $\mathrm{PO}_{4}, 0.2 \mu \mathrm{g} / \mu \mathrm{l}$ poly (dI:dC), $0.2 \mu \mathrm{g} / \mu \mathrm{l} \mathrm{BSA}$, and $95 \mathrm{ng}$ DIG-labeled DNA probe. Protein was added in concentrations ranging from 0.6 to $3.0 \mu \mathrm{g}$ in increments of $0.6 \mu \mathrm{g}$. Reactions were incubated at $16^{\circ} \mathrm{C}$ for $30 \mathrm{~min}$. NP-40 was added to each reaction mixture at a concentration of $0.1 \%$ prior to separation on a pre-run $5 \%$ polyacrylamide gel. Gels were stained with SYBR green and then transferred onto Hybond $\mathrm{N}+$ (Amersham Biosciences, Piscataway, NJ). Probing and detection of DIG-labeled DNA was performed with the DIG Nucleic Acid Detection Kit according to the manufacturer's protocol for colorimetric detection.

\section{Acknowledgements}

We thank Andrea McCarthy for assistance with the siderophore production assays and Mauricio Barajas for assistance with recombinant protein expression. This research was supported in part by the Office of Science (BER), U.S. Department of Energy, Grant No. DE-FG02-06ER64163, to DKT.

\section{Author details}

'Department of Biological Sciences, Purdue University, West Lafayette, IN 47907, USA. ${ }^{2}$ Department of Basic Sciences, College of Veterinary Medicine, Mississippi State University, Mississippi State, MS 39762, USA. ${ }^{3}$ Center for Environmental Biotechnology, University of Tennessee, Knoxville, TN 37996, USA.

\section{Authors' contributions}

KLH carried out the expression and partial purification of the recombinant SO2426 and SO2426sh proteins, performed electrophoretic mobility shift assays and siderophore production measurements, and wrote the majority of the manuscript. XFW generated the multiple sequence alignment and phylogenetic tree for SO2426 orthologs in Shewanella, identified the predicted recognition site for $\mathrm{SO} 2426$ binding, and contributed to the production of the manuscript. WW constructed the vectors for recombinant SO2426 and SO2426sh expression. DKT conceived the study, helped to supervise the experiments, and participated in the writing of the manuscript. All authors read and approved the final manuscript.

Received: 23 August 2010 Accepted: 31 May 2011

Published: 31 May 2011

\section{References}

1. Raivio TL, Silhavy TJ: Periplasmic stress and ECF sigma factors. Annu Rev Microbiol 2001, 55:591-624.

2. West $A H$, Stock AM: Histidine kinases and response regulator proteins in two-component signaling systems. Trends Biochem Sci 2001, 26:369-376.

3. Ulrich LE, Koonin EV, Zhulin IB: One-component systems dominate signal transduction in prokaryotes. Trends Microbiol 2005, 13:52-56.

4. Gueriri I, Cyncynatus C, Dubrac S, Arana AT, Dussurget O, Msadek T: The DegU orphan response regulator of Listeria monocytogenes autorepresses its own synthesis and is required for bacterial motility, virulence and biofilm formation. Microbiology 2008, 154:2251-2264.

5. Delany I, Spohn G, Rappuoli R, Scarlato V: Growth phase-dependent regulation of target gene promoters for binding of the essential orphan response regulator HP1043 of Helicobacter pylori. J Bacterio/ 2002, 184:4800-4810.

6. Hong E, Lee HM, Ko H, Kim DU, Jeon BY, Jung J, Shin J, Lee SA, Kim Y, Jeon $\mathrm{YH}$, et al: Structure of an atypical orphan response regulator protein 
supports a new phosphorylation-independent regulatory mechanism. J Biol Chem 2007, 282:20667-20675.

7. Pan X, Ge J, Li M, Wu B, Wang C, Wang J, Feng Y, Yin Z, Zheng F, Cheng G, et al: The orphan response regulator CovR: a globally negative modulator of virulence in Streptococcus suis serotype 2. J Bacteriol 2009, 191:2601-2612.

8. Ulijasz AT, Andes DR, Glasner JD, Weisblum B: Regulation of iron transport in Streptococcus pneumoniae by RitR, an orphan response regulator. J Bacteriol 2004, 186:8123-8136.

9. Echave P, Tamarit J, Cabiscol E, Ros J: Novel antioxidant role of alcohol dehydrogenase E from Escherichia coli. J Biol Chem 2003, 278:30193-30198.

10. Gao H, Wang X, Yang ZK, Palzkill T, Zhou J: Probing regulon of ArcA in Shewanella oneidensis MR-1 by integrated genomic analyses. BMC Genomics 2008, 9:42.

11. Andrews SC, Robinson AK, Rodriguez-Quinones F: Bacterial iron homeostasis. FEMS Microbiol Rev 2003, 27:215-237.

12. Wan XF, Verberkmoes NC, McCue LA, Stanek D, Connelly H, Hauser LJ Wu L, Liu X, Yan T, Leaphart A, et al: Transcriptomic and proteomic characterization of the Fur modulon in the metal-reducing bacterium Shewanella oneidensis. J Bacteriol 2004, 186:8385-8400.

13. Yang Y, Harris DP, Luo F, Wu L, Parsons AB, Palumbo AV, Zhou J: Characterization of the Shewanella oneidensis Fur gene: roles in iron and acid tolerance response. BMC Genomics 2008, 9(Suppl 1):S11.

14. Yang $Y$, Harris DP, Luo F, Xiong $W$, Joachimiak $M, W u$, Dehal $P$, Jacobsen J, Yang Z, Palumbo AV, et al: Snapshot of iron response in Shewanella oneidensis by gene network reconstruction. BMC Genomics 2009, 10:131.

15. Brown SD, Thompson MR, Verberkmoes NC, Chourey K, Shah M, Zhou J, Hettich RL, Thompson DK: Molecular dynamics of the Shewanella oneidensis response to chromate stress. Mol Cell Proteomics 2006, 5:1054-1071.

16. Thompson MR, VerBerkmoes NC, Chourey K, Shah M, Thompson DK, Hettich RL: Dosage-dependent proteome response of Shewanella oneidensis MR-1 to acute chromate challenge. J Proteome Res 2007, 6:1745-1757.

17. Henne KL, Turse JE, Nicora CD, Lipton MS, Tollaksen SL, Lindberg C, Babnigg G, Giometti CS, Nakatsu CH, Thompson DK, Konopka AE: Global proteomic analysis of the chromate response in Arthrobacter sp. strain FB24. J Proteome Res 2009, 8:1704-1716.

18. Bagchi D, Stohs SJ, Downs BW, Bagchi M, Preuss HG: Cytotoxicity and oxidative mechanisms of different forms of chromium. Toxicology 2002, 180:5-22.

19. Wang CC, Newton A: Iron transport in Escherichia coli: relationship between chromium sensitivity and high iron requirement in mutants of Escherichia coli. J Bacteriol 1969, 98:1135-1141.

20. Chourey K, Thompson MR, Morrell-Falvey J, Verberkmoes NC, Brown SD, Shah M, Zhou J, Doktycz M, Hettich RL, Thompson DK: Global molecular and morphological effects of 24-hour chromium(VI) exposure on Shewanella oneidensis MR-1. Appl Environ Microbiol 2006, 72:6331-6344.

21. Chourey K, Wei W, Wan XF, Thompson DK: Transcriptome analysis reveals response regulator SO2426-mediated gene expression in Shewanella oneidensis MR-1 under chromate challenge. BMC Genomics 2008, 9:395.

22. Martinez-Hackert E, Stock AM: Structural relationships in the OmpR family of winged-helix transcription factors. J Mol Biol 1997, 269:301-312.

23. Konstantinidis $K T$, Serres $M H$, Romine MF, Rodrigues $J$, Auchtung J, McCue LA, Lipton MS, Obraztsova A, Giometti CS, Nealson KH, et al: Comparative systems biology across an evolutionary gradient within the Shewanella genus. Proc Natl Acad Sci USA 2009, 106:15909-15914.

24. Hau HH, Gralnick JA: Ecology and biotechnology of the genus Shewanella. Annu Rev Microbiol 2007, 61:237-258.

25. Saltikov CW, Cifuentes A, Venkateswaran K, Newman DK: The ars detoxification system is advantageous but not required for $\mathrm{As}(\mathrm{V})$ respiration by the genetically tractable Shewanella species strain ANA-3. Appl Environ Microbiol 2003, 69:2800-2809.

26. Aguilar-Barajas E, Paluscio E, Cervantes C, Rensing C: Expression of chromate resistance genes from Shewanella sp. strain ANA-3 in Escherichia coli. FEMS Microbiol Lett 2008, 285:97-100.

27. Bencheikh-Latmani R, Obraztsova A, Mackey MR, Ellisman MH, Tebo BM: Toxicity of $\mathrm{Cr}(\mathrm{III})$ to Shewanella sp. strain MR-4 during $\mathrm{Cr}(\mathrm{VI})$ reduction. Environ Sci Technol 2007, 41:214-220.
28. Karpinets TV, Obraztsova AY, Wang Y, Schmoyer DD, Kora GH, Park BH, Serres MH, Romine MF, Land ML, Kothe TB, et al: Conserved synteny at the protein family level reveals genes underlying Shewanella species' cold tolerance and predicts their novel phenotypes. Funct Integr Genomics 10:97-110.

29. Fredrickson JK, Romine MF, Beliaev AS, Auchtung JM, Driscoll ME, Gardner TS, Nealson KH, Osterman AL, Pinchuk G, Reed JL, et al: Towards environmental systems biology of Shewanella. Nat Rev Microbiol 2008, 6:592-603.

30. Bailey $\mathrm{TL}$, Elkan C: Fitting a mixture model by expectation maximization to discover motifs in biopolymers. Proc Int Conf Intell Syst Mol Biol 1994, 2:28-36.

31. Thijs G, Marchal K, Lescot M, Rombauts S, De Moor B, Rouze P, Moreau Y: A Gibbs sampling method to detect overrepresented motifs in the upstream regions of coexpressed genes. J Comput Biol 2002, 9:447-464.

32. Thompson W, Rouchka EC, Lawrence CE: Gibbs Recursive Sampler: finding transcription factor binding sites. Nucleic Acids Res 2003, 31:3580-3585.

33. De Wulf P, McGuire AM, Liu X, Lin EC: Genome-wide profiling of promoter recognition by the two-component response regulator CpxR-P in Escherichia coli. J Biol Chem 2002, 277:26652-26661.

34. Pogliano J, Lynch AS, Belin D, Lin EC, Beckwith J: Regulation of Escherichia coli cell envelope proteins involved in protein folding and degradation by the Cpx two-component system. Genes Dev 1997, 11:1169-1182.

35. Danese PN, Snyder WB, Cosma CL, Davis L, Silhavy TJ: The Cpx twocomponent signal transduction pathway of Escherichia coli regulates transcription of the gene specifying the stress-inducible periplasmic protease, DegP. Genes Dev 1995, 9:387-398.

36. Ruiz N, Silhavy TJ: Sensing external stress: watchdogs of the Escherichia coli cell envelope. Curr Opin Microbiol 2005, 8:122-126.

37. Batchelor E, Walthers D, Kenney LJ, Goulian M: The Escherichia coli CpxACpxR envelope stress response system regulates expression of the porins ompF and ompC. J Bacteriol 2005, 187:5723-5731.

38. De Wulf $\mathrm{P}$, Kwon O, Lin EC: The CpxRA signal transduction system of Escherichia coli: growth-related autoactivation and control of unanticipated target operons. J Bacteriol 1999, 181:6772-6778.

39. Danese PN, Silhavy TJ: CpxP, a stress-combative member of the Cpx regulon. J Bacteriol 1998, 180:831-839.

40. Yamamoto K, Ishihama A: Transcriptional response of Escherichia coli to external copper. Mol Microbiol 2005, 56:215-227.

41. Chourey K, Thompson MR, Shah M, Zhang B, Verberkmoes NC, Thompson DK, Hettich RL: Comparative temporal proteomics of a response regulator (SO2426)-deficient strain and wild-type Shewanella oneidensis MR-1 during chromate transformation. J Proteome Res 2009, 8:59-71.

42. Brown SD, Martin M, Deshpande S, Seal S, Huang K, Alm E, Yang Y, Wu L, Yan T, Liu X, et al: Cellular response of Shewanella oneidensis to strontium stress. Appl Environ Microbiol 2006, 72:890-900.

43. Fennessey CM, Jones ME, Taillefert M, DiChristina TJ: Siderophores are not involved in $\mathrm{Fe}$ (III) solubilization during anaerobic Fe(III) respiration by Shewanella oneidensis MR-1. Appl Environ Microbiol 2010, 76:2425-2432.

44. Macomber L, Imlay JA: The iron-sulfur clusters of dehydratases are primary intracellular targets of copper toxicity. Proc Natl Acad Sci USA 2009, 106:8344-8349.

45. Guedon E, Moore CM, Que Q, Wang T, Ye RW, Helmann JD: The global transcriptional response of Bacillus subtilis to manganese involves the MntR, Fur, TnrA and sigmaB regulons. Mol Microbiol 2003, 49:1477-1491.

46. Myers $C R$, Myers JM: Iron stimulates the rate of reduction of hexavalent chromium by human microsomes. Carcinogenesis 1998, 19:1029-1038.

47. Myers CR, Myers JM, Carstens BP, Antholine WE: Reduction Of Chromium (VI) To Chromium(V) By Human Microsomal Enzymes: Effects Of Iron and Quinones. Toxic Substance Mechanisms 2000, 19:25-51.

48. Luo H, Lu Y, Shi X, Mao Y, Dalal NS: Chromium (IV)-mediated fenton-like reaction causes DNA damage: implication to genotoxicity of chromate. Ann Clin Lab Sci 1996, 26:185-191.

49. Seo J, Gordish-Dressman H, Hoffman EP: An interactive power analysis tool for microarray hypothesis testing and generation. Bioinformatics 2006, 22:808-814.

50. Bailey TL, Gribskov M: Combining evidence using p-values: application to sequence homology searches. Bioinformatics 1998, 14:48-54.

51. Schneider TD, Stephens RM: Sequence logos: a new way to display consensus sequences. Nucleic Acids Res 1990, 18:6097-6100. 
52. Thompson JD, Higgins DG, Gibson TJ: CLUSTAL W: improving the sensitivity of progressive multiple sequence alignment through sequence weighting, position-specific gap penalties and weight matrix choice. Nucleic Acids Res 1994, 22:4673-4680.

53. Stock AM, Robinson VL, Goudreau PN: Two-component signal transduction. Annu Rev Biochem 2000, 69:183-215.

54. Swofford DL: PAUP*: Phylogenic analysis using Parsimony Sinauer, Sunderland, Massachusetts; 1998.

55. Schwyn B, Neilands JB: Universal chemical assay for the detection and determination of siderophores. Anal Biochem 1987, 160:47-56.

doi:10.1186/1471-2180-11-125

Cite this article as: Henne et al: SO2426 is a positive regulator of siderophore expression in Shewanella oneidensis MR-1. BMC Microbiology 2011 11:125.

Submit your next manuscript to BioMed Central and take full advantage of:

- Convenient online submission

- Thorough peer review

- No space constraints or color figure charges

- Immediate publication on acceptance

- Inclusion in PubMed, CAS, Scopus and Google Scholar

- Research which is freely available for redistribution

Submit your manuscript at www.biomedcentral.com/submit 